\title{
Crescimento, sobrevivência e resistência de larvas de tilápia em função da densidade e da suplementação com vitamina $C$
}

\author{
Lima, A.F. ${ }^{1 @}$ e Barbosa, J.M. ${ }^{2}$ \\ 'Empresa Brasileira de Pesquisa Agropecuária. Embrapa Pesca e Aquicultura. Palmas. TO. Brasil. \\ 2Universidade Federal Rural de Pernambuco. Recife. PE. Brasil.
}

\section{PalaVRas ChaVe adicionaIS}

Crescimento heterogêneo.

Invers̃ão sexual.

Oreochromis niloticus.

\section{ADDITIONAL KEYWORDS \\ Heterogeneous growth. \\ Sex inversion. \\ Oreochromis niloticus.}

\section{INFORMACIÓN}

\section{Cronología del artículo.}

Recibido/Received: 19.05.2015

Aceptado/Accepted: 01.02 .2016

On-line: 11.06 .2016

Correspondencia a los autores/Contact e-mail:

adriana.lima@embrapa.br

\section{RESUMO}

Neste experimento, avaliou-se o efeito da densidade de estocagem e da suplementação de vitamina $\mathrm{C}$ na dieta sobre o crescimento, sobrevivência e resistência de larvas de tilápiado-nilo Oreochromis niloticus, durante a fase de inversão sexual. Os indivíduos, mantidos em aquários, foram distribuídos em delineamento inteiramente casualizado e esquema fatorial $3 \times 2$ nas densidades 2, 4 e 6 larvas/L e alimentados com ração comercial suplementada com $500 \mathrm{mg} \mathrm{kg}^{-1}$ de vitamina $C$, perfazendo $850 \mathrm{mg} \mathrm{kg}^{-1}$ ou não, isto é, com $350 \mathrm{mg} \mathrm{kg}^{-1}$, contidos na própria ração comercial. Ao final do experimento foi realizado um teste de exposição ao ar para avaliar o efeito da vitamina $\mathrm{C}$ na resistência das larvas. Os melhores pesos finais ocorreram nos tratamentos que receberam dieta suplementada com vitamina $\mathrm{Ce}$ foram estocados em baixas densidades. $\mathrm{O}$ aumento da densidade influenciou negativamente os pesos no decorrer do experimento, mas teve efeito positivo na sobrevivência e resistência das larvas. A variação de crescimento, tanto em peso quanto em comprimento, foi afetada pela interação entre os fatores avaliados.

\section{Growth, survival and resistance of tilapia larvae as a function of stock density and vitamin C supplementation}

\section{SUMMARY}

This study aimed at analyzing the effect of the stock density and diet vitamin C supplementation over growth, survival rate and resistance of Nile tilapia larvae /Oreochromis niloticus), during the sexual inversion period. Individuals were maintained in aquariums, completely distributed at random following a $3 \times 2$ factorial design, with 2, 4 and 6 larvae/L densities and fed on $500 \mathrm{mg} \mathrm{kg}^{-1}$ vitamin $C$ supplement commercial feed (totaling $850 \mathrm{mg} \mathrm{kg}^{-1}$ ) or in the absence of Vitamin $C$ supplement, that is $350 \mathrm{mg} \mathrm{kg}^{-1}$, in commercial feed. At the end of the experiment, a test of exposure to air evaluated the effect of vitamin $C$ on larvae stress resistance. The best final weights occurred in treatments that received vitamin $\mathrm{C}$ dietary supplementation and were stocked under low densities. The increase in the stocking density negatively affected weight gain during the experiment, but positively affected the survival rate and resistance of the larvae. The variability of growth was affected by the interaction between the factors.

\section{INTRODUÇÃO}

O aumento da produção de tilápia-do-nilo Oreochromis niloticus (Linnaeus, 1758) no Brasil sugere a necessidade da melhoria nas etapas de produção de alevinos, especialmente quando aplicada a técnica de inversão sexual, com o uso de hormônio masculinizante, de forma a disponibilizar alevinos, em quantidade e qualidade, suficientes para atender a demanda do setor.

A espécie apresenta hábito territorialista, com interações agonísticas entre os indivíduos, resultando na variabilidade de crescimento, que não é interessante para o processo produtivo e à comercialização do produto. Este fenômeno é reportado na literatura pelo termo crescimento heterogêneo (CHet).

A variabilidade de crescimento nos indivíduos pode ser reduzida através de manejos realizados durante a fase de crescimento e engorda (Lima, 2008), mas poucos estudos buscam meios para minimizar o fenômeno durante a inversão sexual, visando ofertar alevinos mais homogêneos e com maior valor de mercado. Altas taxas de inversão sexual acontecem em diferentes densidades de estocagem, porém esta influencia o tamanho final do alevino, numa relação 
inversamente proporcional (Sanches e Hayashi, 1999; Saes et al., 2007).

Estudos para avaliar a variabilidade de crescimento dos alevinos durante a inversão sexual submetidos a diferentes densidades de estocagem precisam ser aprofundados, porém é observado que maiores densidades refletem na exacerbação do estresse nos peixes (Iguchi et al., 2003; Barcellos et al., 2004; Lupatsch et al., 2010), o que pode ter efeito secundário sobre o crescimento.

A deficiência de nutrientes nas rações tem agravado essa situação. Estudos recentes comprovam que a utilização da vitamina $C$ em níveis adequados tem apresentado respostas positivas na redução do estresse (Affonso et al., 2007), além de influenciar diretamente o crescimento dos peixes, que têm mostrado alta sensibilidade a dietas deficientes em ácido ascórbico, especialmente nos estágios iniciais de crescimento (Rotta, 2003). Considerando a rápida perda dessa vitamina quando a ração entra em contato com a água, é necessária a utilização de doses maiores da mesma, sobretudo nas rações para larvas, pois as partículas apresentam pequeno tamanho, propiciando maior lixiviação.

Dessa forma, o objetivo do presente estudo foi avaliar o efeito de diferentes densidades de estocagem aliados à suplementação de vitamina $\mathrm{C}$ à dieta $\mathrm{co}$ mercial no crescimento, sobrevivência e resistência ao estresse de larvas de tilápia-do-nilo no período de inversão sexual.

\section{MATERIAL E MÉTODOS}

O experimento foi desenvolvido no Departamento de Pesca e Aquicultura (DEPAq), da Universidade Federal Rural de Pernambuco, entre novembro e dezembro de 2009, com utilização de larvas adquiridas na Estação de Piscicultura Prof. Johei Koike da mesma universidade.

O experimento foi realizado através de um delineamento inteiramente casualizado, em esquema fatorial $3 \times 2$, com três densidades $(2,4$, e 6 larvas/L) e alimentados com ração comercial suplementada com $500 \mathrm{mg} / \mathrm{kg}$ vitamina C (perfazendo $850 \mathrm{mg} / \mathrm{kg}$ ) ou não, isto é, com $350 \mathrm{mg} / \mathrm{kg}$, contidos na própria ração comercial. Sendo um total de seis tratamentos, com três repetições cada um, a saber, 2C, 2S, 4C, 4S, 6C, $6 \mathrm{~S}$ (quando os números correspondem às densidades $\mathrm{e}$ as letras $C=$ suplementada com vitamina $C$ e $S=$ sem suplementação). As densidades utilizadas estão de acordo com os estudos realizados por Vera Cruz e Mair (1994), Sanches et al. (1999), Phelps e Popma (2000) e Saes et al. (2007). O nível de vitamina C utilizado atende ao sugerido por Toyama et al. (2000).

Foram utilizados 18 aquários, com volume útil de sete litros, em sistema estático, com aeração individual. Foram selecionadas larvas com tamanho inferior a $14 \mathrm{~mm}$, conforme sugerido por Phelps e Popma (2000), para se obter taxa de inversão sexual entre 97 e $100 \%$. O período experimental foi de 28 dias, tempo necessário para se completar o processo de inversão sexual, no qual os indivíduos foram expostos às diferentes densidades e receberam alimentação com diferentes concentrações de vitamina $C$.
Tabela I. Composição das rações utilizadas no experimento (Test diets composition).

\begin{tabular}{lcc}
\hline \multirow{2}{*}{ Nutrientes } & \multicolumn{2}{c}{ Dieta } \\
\cline { 2 - 3 } & 350 mg Vit. C & 850 mg Vit. C \\
\hline Proteína bruta (\%) & 45,04 & 44,96 \\
Extrato etéreo (\%) & 10,25 & 9,99 \\
Matéria mineral (\%) & 8,25 & 8,29 \\
Matéria seca (\%) & 94,43 & 94,59 \\
Energia bruta (kcal/kg) & 4685,92 & 4568,67 \\
\hline
\end{tabular}

No início e ao final do experimento foram tomados os pesos e comprimentos dos indivíduos, para determinação do peso e comprimento médio e do coeficiente de variação $(\mathrm{CV}=$ desvio padrão/média do peso ou comprimento $\mathrm{x} 100$ ), utilizado como indicador do crescimento heterogêneo. Os exemplares foram pesados e mensurados individualmente. Utilizou-se balança digital com precisão de quatro casas decimais. Para acompanhamento do ganho em peso ao longo do experimento, foram realizadas pesagens em grupos de indivíduos para cada parcela experimental no $9^{\circ} \mathrm{e}$ $18^{\circ}$ dia.

As variáveis limnológicas: oxigênio, $\mathrm{pH}$, amônia foram monitoradas semanalmente e temperatura diariamente. Os aquários foram sifonados diariamente para remoção de detritos e excretas e a quantidade de água retirada, cerca de $30 \%$ do volume total, foi reposta.

Os animais foram alimentados ad libitum, seis vezes ao dia $(8,10,12,14,16$ e 18 h), com utilização de ração comercial farelada para fases inicias de vida, acrescidas de $60 \mathrm{mg}$ do hormônio masculinizante 17- $\alpha$-metil testosterona para cada quilograma de ração, conforme metodologia proposta por Phelps et al. (2000).

Para se obter a concentração final de $850 \mathrm{mg}$ de vitamina $C$ na dieta suplementada, foi utilizada a suplementação de $500 \mathrm{mg}$ de vitamina C (ácido ascórbico) por quilograma da ração comercial, que, segundo o fabricante, contém $350 \mathrm{mg}$ desta vitamina em sua composição original.

Para inclusão da vitamina na ração comercial foi utilizado como veículo óleo de soja na proporção de $1 \%$, segundo metodologia descrita por Okamura et al. (2007), que observaram atuação do óleo protegendo e aderindo a vitamina $C$ à ração por tempo suficiente para o seu consumo. A ração foi mantida sob refrigeração e em recipiente escuro. A mesma quantidade de óleo de soja foi acrescida à ração sem adição de vitamina, afim de que as larvas recebam dieta com mesma característica nutricional. Foi realizada análise bromatológica da ração, segundo metodologia descrita por Silva e Queiroz (2002), objetivandos-e avaliar a composição final da mesma (tabela I).

Para avaliação da resistência das larvas, foi realizado um teste sobre papel secante conforme Luz e Portella (2005), contudo, foi utilizado maior tempo de exposição ao ar, já que Luz e Portella não encontraram efeitos para exposição por 3 minutos. Os animais foram retirados dos aquários, colocados em papel secante e expostos ao ar por 5 minutos. Posteriormente, o papel 
Tabela II. Valor do teste F e valores médios do peso $(\mathrm{g})$ ( \pm desvio padrão) aos 9, 18 e 28 dias, coeficiente de variação do peso (CVP), comprimento final, coeficiente de variação do comprimento (CVC $(\%)$ ), sobrevivência final (SF $(\%)$ ) e mortalidade após o teste de resistência (MTR $(\%)$ ) (F value and average ( \pm standard deviation) for weight at 9,18 and 28 day production $(\mathrm{g})$, weight and length coefficient of variation (\%), final survival rate (\%), and mortality rate after resistance test (\%)).

\begin{tabular}{|c|c|c|c|c|c|c|c|c|}
\hline \multirow[b]{2}{*}{ Estatística } & \multicolumn{8}{|c|}{ F valor } \\
\hline & Peso (9 dias) & Peso (18 dias) & Peso (28 dias) & CVP & Comprimento & CVC & SF & MTR \\
\hline Suplementação de vitamina C (S) & $0,39 \mathrm{~ns}$ & $3,86 \mathrm{~ns}$ & $34,46^{* * *}$ & $2,51 \mathrm{~ns}$ & $10,45^{\star *}$ & $0,79 \mathrm{~ns}$ & $2,39 \mathrm{~ns}$ & 0,003 ns \\
\hline Densidade (D) & $12,02^{* *}$ & $11,76^{* *}$ & $16,13^{* * *}$ & $0,79 \mathrm{~ns}$ & $2,69 \mathrm{~ns}$ & $1,06 \mathrm{~ns}$ & $9,97^{* *}$ & $4,42^{*}$ \\
\hline Interação S x D & $0,29 \mathrm{~ns}$ & $1,86 \mathrm{~ns}$ & $2,23 \mathrm{~ns}$ & $4,04^{*}$ & $0,12 \mathrm{~ns}$ & $9,78^{* *}$ & $0,15 \mathrm{~ns}$ & $0,90 \mathrm{~ns}$ \\
\hline Tratamentos & \multicolumn{8}{|c|}{ Médias para suplementação de vitamina C } \\
\hline Com & $0,06 \pm 0,01$ & $0,09 \pm 0,02$ & $0,17 \pm 0,03^{a}$ & $70,0 \pm 18,3$ & $1,71 \pm 0,15^{a}$ & $25,5 \pm 5,2$ & $53 \pm 20$ & $77 \pm 26$ \\
\hline \multirow[t]{2}{*}{ Sem } & $0,06 \pm 0,01$ & $0,08 \pm 0,02$ & $0,12 \pm 0,02^{b}$ & $81,4 \pm 17,1$ & $1,54 \pm 0,08 \underline{b}$ & $27,4 \pm 7,4$ & $44 \pm 17$ & $77 \pm 23$ \\
\hline & \multicolumn{8}{|c|}{ Médias para densidade } \\
\hline 2 & $0,07 \pm 0,01^{a}$ & $0,10 \pm 0,01^{a}$ & $0,17 \pm 0,02^{\mathrm{a}}$ & $69,3 \pm 10,9$ & $1,69 \pm 0,14$ & $25,5 \pm 4,4$ & $30 \pm 11^{a}$ & $97 \pm 4^{a}$ \\
\hline 4 & $0,06 \pm 0,01^{b}$ & $0,08 \pm 0,01^{b}$ & $0,15 \pm 0,03^{a}$ & $78,83 \pm 23,5$ & $1,63 \pm 0,14$ & $28,6 \pm 8,9$ & $54 \pm 15^{b}$ & $71 \pm 25^{\mathrm{ab}}$ \\
\hline 6 & $0,05 \pm 0,00^{b}$ & $0,08 \pm 0,02^{b}$ & $0,13 \pm 0,05^{b}$ & $78,92 \pm 19,4$ & $1,54 \pm 0,13$ & $25,3 \pm 5,3$ & $62 \pm 13^{b}$ & $63 \pm 22^{b}$ \\
\hline
\end{tabular}

Médias seguidas por letras diferentes na vertical diferem significativamente, pelo teste de Tukey $p<0,05 .{ }^{*} p<0,05 ;{ }^{* *} p<0,01 ;{ }^{* * *} p<0,001$; ns= não significativo.

secante foi mergulhado de volta nos aquários e as larvas que permaneceram aderidas foram cuidadosamente retiradas. A sobrevivência foi observada duas e 24 horas após a exposição.

Para análise estatística, os testes de Shapiro-Wilk e Bartlett foram utilizados para verificar se os dados atendiam as pressuposições de normalidade e homogeneidade, respectivamente. Posteriormente, foi realizada a analise de variância para esquema fatorial e quando não houve efeito significativo para a interação, realizou-se o teste de Tukey para comparar as médias dos tratamentos. Já quando houve interação, foi realizado desdobramento do fatorial e as médias foram comparadas pelo teste de Tukey, a 5\% de probabilidade. As análises foram realizadas pelo software estatístico R (R Core Team, 2015).

\section{RESULTADOS E DISCUSSÃO}

A concentração de oxigênio dissolvido na água $(6,17 \pm 0,99 \mathrm{mg} / \mathrm{L})$, a temperatura $\left(26,5 \pm 0,14^{\circ} \mathrm{C}\right)$, o $\mathrm{pH}$ $(7,33 \pm 0,13)$ e a amônia total $(1,30 \pm 0,88 \mathrm{mg} / \mathrm{L})$ não apresentaram grandes variações e mantiveram-se dentro dos padrões aceitáveis para a espécie (Popma e Lovshin, 1994), o que sugere a não influência destas variáveis nos resultados obtidos.

Com respeito ao crescimento, no início do experimento, os peixes apresentavam pesos similares. Não houve diferença significativa considerando a interação entre a densidade e suplementação de vitamina $C$ na ração para sobrevivência final, peso e comprimento ao longo e ao final do experimento (tabela II).

Contudo foram observadas diferenças significativas no peso quando se avaliou a influência dos fatores densidade e suplementação de vitamina $C$ isoladamente, sendo que até o 18o dia de experimento, o fator densidade responsável por esta diferença entre os pesos (tabela II), com uma relação inversamente proporcional. Resultado semelhante ao encontrado por Saes et al.
(2007) que observaram que quanto maior a densidade de estocagem, menor o ganho de peso de larvas de tilápia durante a fase de inversão sexual, concluindo que isso ocorre devido à competição por espaço, alimento e interação social. Este efeito da densidade sobre o crescimento de peixes foi observado também por diversos autores, como Souza-Filho e Cerqueira (2003), em juvenis de robalo-flecha Centropomus undecimali (Bloch, 1792), Brandão e colaboradores (2004) em juvenis de tambaqui Colossoma macropomum (Cuvier, 1816) e Almeida e Nuñer (2009), em juvenis de mandi-amarelo Pimelodus maculatus Lacepède, 1803. Sanches e Hayashi (1999) observaram a mesma relação entre a densidade e o crescimento para tilápia na fase de inversão, mas só conseguiram perceber o efeito da densidade sobre a diminuição do crescimento a partir da terceira semana de criação, enquanto neste experimento, no nono dia já foi possível observar a diferença de crescimento entre os tratamentos submetidos a diferentes densidades.

A pouca influência da suplementação de vitamina $\mathrm{C}$ na dieta nos primeiros dias pode estar relacionado à transferência de nutrientes, inclusive ácido ascórbico, dos reprodutores para larvas, via vitelo, o que assegura reservas para as larvas nos estágios iniciais de vida, relação estudada por Soliman et al. (1986) e Izquierdo et al. (2001).

A suplementação de vitamina $C$ na dieta também foi responsável pela diferença de peso e comprimento na fase final da inversão, com o grupo de animais alimentados com dieta suplementada com vitamina $C$ apresentando peso e comprimento superior. Esse efeito corrobora os resultados de Affonso et al. (2007), para juvenis de matrinchã (Brycon amazonicus), e Toyama et al. (2000), que encontraram melhor peso, comprimento e sobrevivência quando utilizaram níveis superiores a $800 \mathrm{mg}$ de vitamina $\mathrm{C}$ por $\mathrm{kg}$ em dietas utilizadas na alimentação de larvas de tilápia. Porém, estes autores não avaliaram a influência desta vitamina no coeficiente de variação e resistência das larvas. 
Menor taxa de crescimento nos animais que receberam dieta não suplementada pode estar relacionado à inadequada quantidade de vitamina $C$ nesta dieta, fato que não foi observado na fase inicial de vida das larvas, o que pode ocorrer em função da utilização de reservas endógenas, que não foram suficientes para suprir as necessidades dos animais até o final do experimento. Os pesos finais dos peixes alimentados com dieta suplementada com vitamina C (0,17 g) estão de acordo com o relatado por Popma et al. (1994) para tilápia-do-nilo no final do período de inversão sexual (entre 0,15 e 0,8 g).

A sobrevivência dos peixes não foi afetada pela interação entre a densidade de estocagem e a suplementação de vitamina $C$ na dieta, mas isoladamente pelo fator densidade de estocagem (tabela II), com as menores densidades apresentando menor sobrevivência , relação encontrada também por Jurgensen et al. (1993), para Salvelinus alpinus (Linnaeus, 1758) e Souza-Filho et al. (2003), para juvenis de robalo-flecha Centropomus undecimalis (Bloch, 1792). Esta relação pode ser compreendida pela exacerbação das interações sociais em ambientes com menor densidade de estocagem, resultando ainda em maior variabilidade de crescimento e menor resistência à estressores (Wedemeyer, 1996; Iwama et al., 1997) (tabela II).

$\mathrm{Na}$ avaliação da variabilidade de crescimento, a interação entre os fatores densidade e suplementação de vitamina $C$ na dieta foi significativa (tabela III). Verificou-se um menor coeficiente de variação em peso para animais mantidos na densidade de 4 larvas/L e alimentados com ração suplementada com vitamina $C$. Os demais tratamentos não diferiram entre si. A influência da densidade na variabilidade de crescimento é um tema ainda controverso na literatura (Irwin et al., 1999; Gomes et al., 2000; Petit et al., 2001; Cavero et al., 2003; Souza-Filho et al., 2003; Brandão et al., 2004; Brandão et al., 2005; Saes et al., 2007). Tanto no coeficiente de variação em peso quanto em comprimento, diferenças foram observadas para a densidade de 4 larvas/L. Presume-se que essa densidade não foi suficiente para quebrar a hierarquia social da tilápia. Paralelamente, pode-se perceber uma redução na variabilidade de crescimento dos animais alimentados com dieta suplementada com vitamina $\mathrm{C}$ em detrimento daqueles que receberam dieta não suplementada, resultado de níveis adequados de vitamina $C$ na ração, que pode ter contribuído para a atenuação do estresse causado pela interação social.

Sanches et al. (1999) afirmam que o crescimento desigual é um agravante na larvicultura de tilápia que, em níveis altos, traz como consequência o canibalismo. Neste experimento, o canibalismo pode ter sido responsável pela maior parte da mortalidade observada em todos os tratamentos, uma vez que ao longo do experimento foi observada baixa mortalidade aparente (visualização de larvas mortas) e alta taxa de crescimento heterogêneo em todos os tratamentos, o que resulta em grandes diferenças de tamanho entre os indivíduos, facilitando o canibalismo. Adicionalmente, uma maior mortalidade foi observada nas menores densidades de estocagem, o que pode também ser resultado de uma exposição a maiores níveis de estresse,
Tabela III. Valores médios ( \pm desvio padrão) do coeficiente de variação do peso (CVP) e comprimento (CVC) na interação entre os fatores densidade de estocagem e suplementação de vitamina $C$ na dieta (Density and vitamin C supplementation on diet interaction average values ( \pm standard deviation) for weight and length coefficient of variation).

\begin{tabular}{lccc}
\hline Densidade (larva/L) & 2 & 4 & 6 \\
\hline Suplementação de vitamina C & \multicolumn{3}{c}{ CVP } \\
\hline Com & $69 \pm 12,9^{\text {Aa }}$ & $59 \pm 5,6^{\mathrm{Ba}}$ & $82 \pm 27,1^{\text {Aa }}$ \\
Sem & $70 \pm 11,4^{\text {Aa }}$ & $99 \pm 12,5^{\text {Aa }}$ & $76 \pm 13,2^{\mathrm{Aa}}$ \\
\hline \multicolumn{4}{c}{ CVC } \\
\hline Com & $26 \pm 6^{\mathrm{Aa}}$ & $21 \pm 2^{\mathrm{Ba}}$ & $28 \pm 5^{\text {Aa }}$ \\
Sem & $24 \pm 4^{\text {Ab }}$ & $35 \pm 6^{\text {Aa }}$ & $21 \pm 1^{\text {Ab }}$ \\
\hline
\end{tabular}

Médias seguidas por letras iguais (,$B$ na vertical e a,b,c na horizontal) não diferem significativamente pelo teste de Tukey $(p<0,05)$.

resultante de interações agonísticas entre os indivíduos, que são mais intensas em baixas densidades de estocagem (Wedemeyer, 1996; Iwama et al., 1997).

A mortalidade dos animais após o teste de resistência foi influenciada apenas pelo fator densidade (tabela II). Sakakura et al. (1998), expondo juvenis de Seriola quinqueradiata Temminck and Schlegel, 1845 alimentados com dieta contendo diferentes níveis de vitamina $C(0,400,1000$ e $2000 \mathrm{mg} / \mathrm{kg}$ dieta) ao teste de exposição ao ar, não encontraram diferenças significativa na mortalidade após o teste entre os tratamentos. Luz e Portella (2005), avaliando a resistência de larvas e juvenis de trairão Hoplias lacerdae Miranda Ribeiro, 1908 submetidos a teste de exposição ao ar por 3 minutos, não encontraram diferença significativa entre indivíduos de diferentes idades. Estes autores observaram que tratamentos com exposição ao ar inferior a sete minutos não são suficientes para induzir respostas diferenciadas nos animais, contudo, neste estudo, o tempo de 5 minutos já foi suficiente para induzir respostas para larvas de tilápia. Assim como o observado para a sobrevivência final, o fato das menores mortalidades no teste de resistência estar relacionada a maiores densidades pode sugerir que o aumento da densidade reduz os confrontos agonísticos em espécies territoriais (Wedemeyer, 1996; Iwama et al., 1997), reduzindo o estresse da submissão e consequentemente seus efeitos sobre a resistência dos animais.

Os animais, logo após o teste, apresentaram perda do equilíbrio hidrostático durante os primeiros minutos após terem sido recolocados no aquário. No entanto, aqueles que resistiram ao teste apresentaram recuperação total após duas horas. A mortalidade dos peixes não se alterou entre duas horas e 24 horas após a exposição dos animais ao ar em papel secante. Por outro lado, Luz et al. (2005) observaram a necessidade de tempos maiores (quatro a oito horas) para recuperação do trairão submetidos a testes de exposição ao ar. Esse fato pode estar relacionado à maior rusticidade da tilápia-do-nilo. Dessa forma, é possível observar que são necessários tempos menores para recuperação da tilápia-do-nilo e, por- 
tanto, menor tempo para a obtenção da sobrevivência final dessa espécie quando submetida a testes de resistência, como o de exposição ao ar.

\section{CONCLUSÃO}

De forma geral, conclui-se que a densidade afeta o crescimento em peso da tilápia ao longo e no final da inversão sexual, enquanto a suplementação de vitamina $C$ na dieta influencia apenas o peso no final do período de inversão sexual. A sobrevivência e a resistência a estressores da larva de tilápia são influenciadas pela densidade. A interação entre a suplementação de vitamina $C$ na dieta e a densidade de estocagem afeta apenas a variabilidade de crescimento em peso e comprimento.

\section{AGRADECIMENTOS}

Ao $\mathrm{CNPq}$ pela bolsa concedida à primeira autora do artigo e ao Laboratório de Nutrição Animal do Departamento de Zootecnica da Universidade Federal Rural de Pernambuco pelas análises realizadas.

\section{BIBLIOGRAFIA}

Affonso, E.G.; Silva, E.C.; Tavares-Dias, M.; Menezes, G.C.; Carvalho, C.S.M.; Nunes, E.S.S.; Ituassú, D.R.; Roubach, R.; Ono, E.A.; Fim, J.D.I. and Marcon, J.L. 2007. Effect of high levels of dietary vitamin $\mathrm{C}$ on the blood responses of matrinxã (Brycon amazonicus). Aquaculture, 147: 383-388.

Almeida, S.C.A. e Nuñer, A.P.O. 2009. Crescimento de Pimelodus maculatus (Actinopterygii, Pimelodidae) estocados em diferentes densidades em tanques-rede. Biotemas, 22: 113-119.

Barcellos, L.J.G.; Kreutz, L.C.; Quevedo, R.M.; Fioreze, I.; Cericato, L.; Soso, A.B.; Fagundes, M.; Conrad, J.; Baldissera, R.K.; Bruschi, A. and Ritter, F. 2004. Nursery rearing of jundia, Rhamdia quelen (Quoy e Gaimard) in cages: cage type, stocking density and stress response to confinement. Aquaculture, 232: 383-394.

Brandão, F.R.; Gomes, L.C.; Chagas, E.C. eAraújo, L.D. 2004. Densidade de estocagem de juvenis de tambaqui durante a recria em tanquesrede. Pesq Agropec Bras, 39: 357-362.

Brandão, F.R.; Gomes, L.C.; Chagas, E.C. Araújo, L.D. e Silva, A.L.F. 2005. Densidade de estocagem de matrinxã (Brycon amazonicus) na recria em tanque-rede. Pesq Agropec Bras, 40: 299-303.

Cavero, B.A.S.; Pereira-Filho, M.; Roubach, R. Ituassú, D.R.; Gandra, A.L. e Crescêncio, R. 2003. Efeito da densidade de estocagem na homogeneidade do crescimento de juvenis de pirarucu em ambiente confinado. Pesq Agropec Bras, 38: 103-107.

Gomes, L.C.; Baldisserotto, B. and Senhorini, J.A. 2000. Effect of stocking density on water quality, survival, and growth of larvae of matrinxã, Brycon cephalus (Characidae), in ponds. Aquaculture, 183: 73-81.

Iguchi, K.; Ogawa, K. and Nagae, M.; ITO, F. 2003. The influence of rearing density on stress response and disease susceptibility of ayu (Plecoglossus altivelis). Aquaculture, 220: 515-523.

Irwin, S.; O'halloran, J. and Fitzgerald, R.D. 1999. Stocking density, growth and growth variation in juvenile turbot, Scophthalmus maximus (Rafinesque). Aquaculture, 178: 77-88.
Iwama, G.K.; Pickering, A.D.; Sumpter, J.P. and Schreck, C.B. (Eds.). 1997. Fish Stress and Health in Aquaculture. University Press. Cambridge. $290 \mathrm{pp}$.

Izquierdo, M.S.; Fernández-Palaciosa, H. and Taconb, A.G.J. 2001. Effect of broodstock nutrition on reproductive performance of fish. Aquaculture, 197: 25-42.

Jurgensen, E.H.; Christiansen, J.S. and Jobling, M. 1993. Effect of stocking density on food intake, growth performanc e and oxygen consumption in Arctic charr (Salvelinus alpinus). Aquaculture, 110: 191-204.

Lima, A.F. 2008. Crescimento heterogêneo em tilápias cultivadas em tanques-rede e submetidas a classificações periódicas. Repesca, 3: 98-101.

Lupatsch, I.; Santos, G.A.; Schrama, J.W. and Verreth, J.A.J. 2010. Effect of stocking density and feeding level on energy expenditure and stress responsiveness in European sea bass Dicentrarchus labrax. Aquaculture, 298: 245-250.

Luz, R.K. and Portella, M.C. 2005. Tolerance to the air exposition test of Hoplias lacerdae larvae and juvenile during its initial development. Braz Arch Biol Technol, 48: 567-573.

Okamura, D.; Araújo, F.G.; Logato, P.V.R.; Murgas, L.D.S.; Freitas, R.T.F.e Araújo, R.V. 2007. Efeito da vitamina C sobre o hematócrito e glicemia de alevinos de tilápia-do-nilo (Oreochromis niloticus) em transporte simulado. Arq Bras Med Vet Zootec, 59: 883-888.

Petit, G.; Beauchad, M. and Buisson, B. Density effects on food intake and growth of largemouth bass (Micropterus salmoides). Aquac Res, 32: $492-495$.

Phelps, R.P. and Popma, T.J. 2000. Sex reversal of tilapia. In: Costa-Pierce, B.A. and Rakocy, J.E. (Eds.) Tilapia Aquaculture in the Americas. Louisiana. The World Aquaculture Society. United States. 34-59.

Popma, T.J. and Lovshin, L.L. 1994. Worldwide prospects for commercial production of tilapia. Auburn University. Auburn. 40 pp.

Silva, D.J. e Queiroz, A.C. 2002. Análise de alimentos: Métodos químicos e biológicos. Universidade Federal de Viçosa. Viçosa. 235 pp.

Rotta, M.A. 2003. Utilização do ácido ascórbico (vitamina C) pelos peixes. Embrapa. Brasília. 54 pp.

Saes, L.A.; Tachibana, L.; Leonardo, A.F.G. e Corrêa, C.F. 2007. Densidade de estocagem durante a fase de reversão sexual da tilápia-do-nilo. In: Seminário de Iniciação Científica do Instituto de Pesca, 2. São Paulo. Anais... Instituto de Pesca. São Paulo. p. 20.

Sakakura, Y.; Koshio, S.; Lida, Y.; Tsukamoto, K.; Kida, T. and Blom, J.H. 1998. Dietary vitamin $C$ improves the quality of yellowtail (Seriola quinqueradiata) seedlings. Aquaculture, 161: 427-436.

Sanches, L.E.F. e Hayashi, C. 1999. Densidade de estocagem no desempenho de larvas de tilápia-do-nilo (Oreochromis niloticus L.), durante a reversão sexual. Acta Sci Anim Sci, 21: 610-625.

Soliman, A.K.; Jauncey, K. and Roberts, R.J. 1986. The effect of dietary ascorbic acid supplementation on hatchability, survival rate and fry performance in Oreochromis mossambicus (Peters). Aquaculture, 59: 197-208.

Souza-Filho, J.J. e Cerqueira, V.R. 2003. Influência da densidade de estocagem no cultivo de juvenis de robalo-flecha mantidos em laboratório. Pesq Agropec Bras, 38: 1317-1322.

Toyama, G.N.; Corrente, J.E. e Cyrino, J.E.P. Suplementação de vitamina $\mathrm{C}$ em rações para reversão sexual da tilápia-do-nilo (Oreochromis niloticus). Sci Agric, 57: 221-228.

Vera Cruz, E.M. and Mair, G.C. Conditions for effective androgen sex reversal in Oreochromis niloticus (L). Aquaculture, 122: 237-248.

Wedemeyer, G.A. 1996. Physiology of fish in intensive culture systems. Chapman \& Hall. New York. 232 pp. 
